\title{
Urinary and Blood MicroRNA-126 and -770 are Potential Noninvasive Biomarker Candidates for Diabetic Nephropathy: a Meta-Analysis
}

\author{
Sungjin Park ${ }^{a, b}$ SeongRyeol Moon ${ }^{c}$ Kiyoung Lee ${ }^{d, e} \quad$ Ie Byung Park ${ }^{d, e}$ \\ Dae Ho Lee ${ }^{c, d, e}$ Seungyoon Nam ${ }^{a, b, c, f}$
}

\begin{abstract}
aDepartment of Genome Medicine and Science, Gachon University College of Medicine, Incheon; ${ }^{\mathrm{b}}$ Gachon Institute of Genome Medicine and Science, Gachon University Gil Medical Center, Incheon; 'Department of Health Sciences and Technology, Gachon Advanced Institute for Health Sciences and Technology, Gachon University, Incheon; 'Department of Internal Medicine, Gachon University Gil Medical Center, Incheon; 'Department of Internal Medicine, Gachon University College of Medicine, Incheon; ' Department of Life Sciences, Gachon University, Seongnam, Korea
\end{abstract}

\section{Key Words}

Diabetes mellitus $•$ Diabetic nephropathy $\bullet$ MicroRNAs $・$ Biomarkers

\begin{abstract}
Background/Aims: Diabetic nephropathy (DN), a major diabetic microvascular complication, has a long and growing list of biomarkers, including microRNA biomarkers, which have not been consistent across preclinical and clinical studies. This meta-analysis aims to identify significant blood- and urine-incident microRNAs as diagnostic/prognostic biomarker candidates for DN. Methods: PubMed, Web of Science, and Cochrane Library were searched from their earliest records through 12th Dec 2016. Relevant publications for the metaanalysis included (1) human participants; (2) microRNAs in blood and urine; (3) DN studies; and (4) English language. Four reviewers, including two physicians, independently and blindly extracted published data regarding microRNA profiles in blood and/or urine from subjects with diabetic nephropathy. A random-effect model was used to pool the data. Statistical associations between diabetic nephropathy and urinary or blood microRNA expression levels were assessed. Results: Fourteen out of 327 studies ( $n=2,747$ patients) were selected. Blood or urinary microRNA expression data of diabetic nephropathy were pooled for this analysis. The hsa-miR-126 family was significantly (OR: 0.57; 95\% CI: 0.44-0.74; p-value < 0.0001) downregulated in blood from patients with diabetic kidney disease, while its urinary level was upregulated (OR: 2931.12; 95\% CI: 9.96-862623.21; p-value = 0.0059). The hsa-miR-770 family microRNA were significantly (OR: 10.24; 95\% CI: 2.37-44.25; $\mathrm{p}$-value $=0.0018$ ) upregulated in both blood and urine from patients with diabetic nephropathy. Conclusions: Our metaanalysis suggests that hsa-miR-126 and hsa-miR-770 family microRNA may have important diagnostic and pathogenetic implications for DN, which warrants further systematic clinical studies.

Dae Ho Lee

and Seungyoon Nam
Department of Internal Medicine, Gachon University Gil Medical Center \& College of

Medicine 774 beongil 21, Namdongdae-ro, Namdong-gu, Incheon, 21565 (Korea)

E-Maildrhormone@naver.com,nams@gachon.ac.kr 


\section{Cellular Physiology Cell Physiol Biochem 2018;46:1331-1340

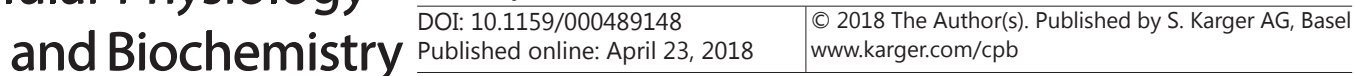 Park et al.: A Meta-Analysis of MiRNAs in Diabetic Nephropathy}

\section{Introduction}

Diabetes mellitus (DM) has a significant societal and economic burden. This disease may progress into numerous complications, influenced by impaired blood glucose control, disease duration, and genetic factors [1]. In 2015, the International Diabetes Federation estimated that 442 million (uncertainty interval: 340-536 million) people aged 20-79 years suffer from diabetes, with a global economic cost of $\$ 673$ billion USD. By 2040, diabetes mellitus incidence is predicted to rise to 642 million (uncertainty interval: 521-829 million) [2]. The 2015 mortality from diabetes mellitus was 5.0 million deaths; that number is predicted to increase by $34 \%$ in 2030 [3]. These tremendous increases in diabetes mellitus incidence (now affecting 1 of 11 persons) are believed to be due to now-endemic obesity and sedentary lifestyles [1].

In addition, diabetes is responsible for approximately 50\% of cases of incident endstage renal disease (ESRD) [4]. The societal burden of both diabetic nephropathy (DN) and ESRD is enormous, both medically and economically [5, 6]. Among various clinical factors, the major predictor of the development and progression of DN and ESRD is albuminuria [7]. Recently, the list of biomarkers of diabetic nephropathy continues to grow, including not only non-classical protein markers, but also genetic and epigenetic markers [8-10]. Also, intensive research has sought to identify biomarkers of diabetic kidney disease (DKD) progression. However, the performance of those markers for predicting DN or ESRD progression has been insufficient compared to the "gold standard" clinical measurements, estimated glomerular filtration rate (eGFR) and urine albumin-creatinine ratio (UACR) [11]. In particular, circulating blood or urinary microRNAs have been proposed to be novel tools for the diagnosis and prognosis of diabetes $[8,9]$. Although various studies have implicated circulating microRNAs as biological markers (and even signal regulators) of DKD, none have been reproducibly shown to associate with diabetes mellitus, nor its renal complications [12]. Additionally, meta-analyses in association with DN and microRNA expression levels have yet to be assessed.

There have been growing lists of microRNA markers for diabetes and its macrovascular and microvascular complications [12]. Furthermore, various clinical studies have shown variable lists of blood or urinary microRNA markers for DN, probably due to small sample sizes, different study designs, the growing list of known microRNAs, and different stages of DN [13-16].

Awaiting the larger and more concrete data on microRNA markers for DN via large and well-designed clinical trials, we aimed to examine the relationship between blood or urinary microRNAs and DN by conducting a meta-analysis of pre-existing clinical data on microRNAs and DN.

\section{Materials and Methods}

\section{Data Sources and Search Strategy}

The databases searched included PubMed, Web of Science, and Cochrane Library, from their earliest records through 12th Dec 2016. For microRNAs associated with DN, we used a combination of keyword terms: diabetes and "miRNA or microRNA" and "microvascular or nephropathy or neuropathy or retinopathy". To secure as many publications as possible, we expanded our search terms to "microvascular" and related terms. We restricted the results to human studies, and references of identified review articles were additionally studied. If necessary, we contacted the corresponding authors to acquire missing information.

Studies were included using the following criteria: (1) those conducted on human subjects relating to the search terms; (2) DN-related studies; (3) microRNA expression results, as exclusively determined by reverse transcription with quantitative PCR (qPCR); and (4) written in English. We excluded studies that 


\section{Cellular Physiology Cell Physiol Biochem 2018;46:1331-1340

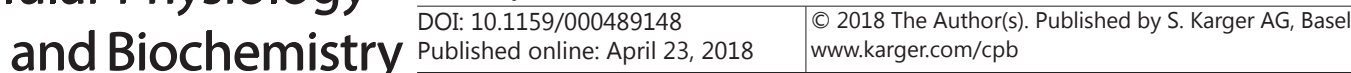

Table 1. Characteristics of Studies

\begin{tabular}{|c|c|c|c|c|c|c|c|c|}
\hline Source & Country & Diabetes & Origin & Exosomes & No. of participants & Mean age, $y$ & Mean diabetes duration, $y$ & Mean HbA1c, \% \\
\hline Al-Kafaji et al. [17] 2016 & Kingdom of Bahrain & Type 2 diabetes & Blood & No & 152 & 60.87 & 16.35 & 7.70 \\
\hline Argyropoulos et al. [18] 2015 & USA & Type 1 diabetes & Urine & No & 27 & 33.13 & 25.23 & 10.00 \\
\hline Argyropoulos et al. [19] 2013 & USA & Type 1 diabetes & Urine & No & 40 & 35.28 & 27.63 & 9.08 \\
\hline Barutta et al. [20] 2017 & Europe & Type 1 diabetes & Blood & No & 457 & 38.45 & 24.50 & 8.30 \\
\hline Barutta et al. [16] 2013 & Italy & Type 1 diabetes & Urine & Yes & 24 & 57.95 & 32.25 & 8.00 \\
\hline Bijkerk et al. [21] 2015 & Netherlands & Type 1 diabetes & Blood & No & 11 & 44.30 & 33.90 & 8.70 \\
\hline Garcia et al. [22] 2015 & $\begin{array}{l}\text { Spain } \\
\text { Span }\end{array}$ & Type 1 diabetes & Blood & No & 114 & 43.20 & 16.86 & 7.74 \\
\hline $\begin{array}{l}\text { Jia et al. [23] } 2016 \\
\text { als }\end{array}$ & China & Type 2 diabetes & Urine & Yes & 90 & 57.30 & 8.05 & 9.47 \\
\hline Liu et al. [24] 2014 & China & Type 2 diabetes & Urine & No & 263 & 50.57 & - & 6.73 \\
\hline Ma et al. [25] 2016 & China & Type 2 diabetes & Blood & No & 591 & 54.12 & 8.26 & 7.73 \\
\hline Pezzolesi et al. [26] 2015 & USA & Type 1 diabetes & Blood & No & 116 & 36.33 & 23.73 & 9.40 \\
\hline $\begin{array}{l}\text { Rezk et al. [27] } 2016 \\
\text { R }\end{array}$ & Egypt & Type 2 diabetes & Blood & No & 286 & 46.73 & 20.15 & $\begin{array}{l}6.90 \\
6.90\end{array}$ \\
\hline Wang et al. [28] 2016 & China & Type 2 diabetes & Blood & No & 276 & 50.10 & 3.45 & 8.47 \\
\hline Zampetaki et al. [29] 2016 & Worldwide & Type 1 diabetes & Blood & No & 300 & 30.33 & 9.03 & 9.10 \\
\hline
\end{tabular}

(1) assessed non-relevant outcomes; (2) did not include qPCR-measured microRNA expression; (3) were qualitative in nature, e.g., case reports, comments, review articles, or guidelines; and (4) were based on animal or in vitro (preclinical) models (Fig. 1).

\section{Study Selection, Data Extraction, and Quality Assessment}

Our initial search produced 327 records. As shown in Fig. 1, application of our inclusion and exclusion criteria resulted in a final total of 14 articles [16-29] for meta-analysis. From these 14, we extracted (1) microRNA expression levels; (2) DN descriptions; (3) study methods; (4) sample origins; (5) type 1 or 2 diabetes mellitus; (6) microRNA expression measurement; (7) the number of participants; and (8) demographics of the participants, including mean age, gender, diabetes duration, and glycated hemoglobin level (Table 1, (for all online suppl. material, see www.karger.com/doi/ 10.1159/000489148) Suppl. Tables S1 and S2). Quality of the 14 individual studies was assessed by following the Cochrane 'Risk of Bias' Tool (see online suppl. material, Suppl. Table S3). Discrepancy for selection was resolved by consensus.

\section{Data Synthesis and Analysis}

For our analysis, we assigned DN, macroalbuminuria, microalbuminuria, and rapid ESRD to distinct groups of patients with DN complications (henceforth, "case groups"). Healthy controls and patients having normoalbuminuria, no diabetes complications, and no other signs of chronic kidney disease were assigned to distinct groups (henceforth, "control groups"). The 14 studies had different effect size measurements, which were transformed to log odds ratios (natural logarithm of odds ratio) and their standard errors.

Following Cochrane meta-analysis guidelines [30], we obtained log odds ratio (henceforth, lnOR) and standard error of InOR (henceforth, $\mathrm{SE}_{\mathrm{InOR}}$ ) in a miRNA for each study. We took $\operatorname{lnOR}$ and $\mathrm{SE}_{\operatorname{lnOR}}$ as input in the R meta-analysis package, "meta".

When an odds ratio (OR) and its 95\% confidential interval (CI) were given in a study, the following equations were used to convert $\operatorname{lnOR}$ and $\mathrm{SE}_{\mathrm{InOR}}$.

$$
\begin{aligned}
& \text { Estimate of effect }=\ln (O R) \\
& S E_{\text {lnOR }}=\frac{(\ln (\text { upper limit of } C I))-(\ln (\text { lower limit of } C I))}{3.92}
\end{aligned}
$$

When OR and p-value $\alpha$ were given in a study, $\ln \mathrm{OR}$ and its $\mathrm{SE}_{\mathrm{InOR}}$ were obtained by using the equations.

$$
\begin{aligned}
& \text { Estimate of effect }=\ln (O R) \\
& S E_{\text {lnOR }}=\frac{\text { Estimate of effect }}{Z}
\end{aligned}
$$

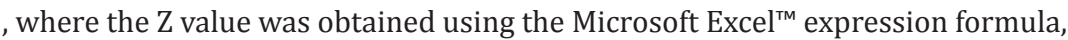

"=ABS(NORMSINV $(\alpha / 2))$ ).

When means and standard deviations for two groups were given in a study, the following steps were used to obtain $\operatorname{lnOR}$ and its $\mathrm{SE}_{\text {lnoR }}$. A pooled standard deviation $\left(\mathrm{S}_{\mathrm{p}}\right)$ and Cohen's d (henceforth, d) were calculated to convert to $\operatorname{lnOR}$. 


$$
S_{p}=\frac{\sqrt{\left(\left(N_{\text {case }}-1\right) \times \sigma_{\text {case }}^{2}+\left(N_{\text {control }}-1\right) \times \sigma_{\text {control }}^{2}\right)}}{\sqrt{\left(N_{\text {case }}+N_{\text {control }}-2\right)}}
$$

, where $N_{i}, i \in\{$ control,case $\}$ isthenumber of samplesingroupiand $\sigma_{i}, i \in\{$ control,case $\}$ is a standard deviation of group $i$. In case SEs or interquartile ranges were given instead of standard deviations, $\sigma_{\mathrm{i}}$ was obtained from the following equations.

$$
\sigma_{i}=\sqrt{N_{i}} \times S E_{i}
$$

or

$$
\sigma_{i}=\frac{Q 3_{i}-Q 1_{i}}{1.35}
$$

, where $S E_{i}, i \in\{$ control, case $\}$ is the $\mathrm{SE}$ of group $i$, and $\mathrm{Q}_{\mathrm{i}}$ and $\mathrm{Q}_{\mathrm{i}}$ are the third quartile and the first quartile in group $i$, respectively.

Then, $\mathrm{d}$ was obtained by the following equation.

$$
d=\frac{\left(\mu_{\text {case }}-\mu_{\text {control }}\right)}{S_{p}}
$$

, where $\mu_{i}, i \in\{$ control, case $\}$ is a mean of group $i$. Finally, lnOR was obtained from the following.

$$
\ln O R=d \times \frac{\pi}{\sqrt{3}}
$$

The $\mathrm{SE}_{\text {InoR }}$ was converted from variance of $\mathrm{d}\left(\mathrm{V}_{\mathrm{d}}\right)$.

$$
\begin{aligned}
& V_{d}=\frac{1}{N_{\text {case }}}+\frac{1}{N_{\text {control }}}+\frac{d^{2}}{2 \times\left(N_{\text {case }}+N_{\text {control }}\right)} \\
& S E_{\text {lnoR }}=\sqrt{V_{d} \times \frac{\pi^{2}}{3}}
\end{aligned}
$$

For sensitivity analysis, we applied a one-study-removed approach to assess the contribution of each study to the pooled effect size. $I^{2}$ was considered a criterion of heterogeneity analysis, in random effect models. We also used a trim-and-fill method [31], to assess the robustness of the effect model, and/or the need to assess publication bias. We also performed the Egger test and examined the resulting funnel plots to identify publication bias [31]. We carried out each of these analyses separately for each microRNA family. All analyses were performed using the "meta" package [32], version 4.8-1, in R.

\section{Results}

\section{Literature Search Results, Study Characteristics, Measurements and Quality}

Our search of the PubMed, Web of Science, and Cochrane Library databases led to the retrieval of 327 studies. Of these, 14 case-control studies met our inclusion and exclusion criteria (Table 1, Fig. 1, (see online suppl. material) Suppl. Table S2), including a total of 2, 747 subjects (mean sample size, 196; range: 11-591 samples), with a mean age of 46.5 years (range: 30.3-60.9 years) and a mean diabetes duration of 19.1 years (range: 3.5-33.9 years). Details of our inclusion criteria for albuminuria and diabetes mellitus, in the 14 studies, are described (see online suppl. material) in Suppl. Table S1. 
In 11 of the 14 studies, microRNA expression, as detected by qPCR, was calculated using the $2^{-\Delta \Delta t}$ relative method [33], using internal controls in each experimental group. Two studies $[23,24]$ did not use internal controls in each experimental group, while another study [29] did not describe a method for determining microRNA expression from raw qPCR data. Quality assessment of the 14 selected studies was described (see online suppl. material) in Suppl. Table S3.

\section{Pooled Effects of Diabetic Nephropathy and microRNAs}

In urine and blood samples, hsamiR-126 was significantly associated with DN (OR: 8.09; 95\% CI: 2.97 to 22.05 with p-value < 0.0001; heterogeneity $I^{2}=98 \%$ with $\mathrm{p}$-value $<0.01$ ) (see online suppl. material, Suppl. Fig. S1), though the directions of these associations were opposite between urine and blood microRNAs (Fig. 2A and (see online suppl. material) Suppl. Fig. S2A).

We then performed subgroup analysis of hsa-miR-126 family expression level, according to sample origin. In blood samples, the hsa-miR-126 family was consistently downregulated in case groups compared to control groups (OR 0.57; 95\% CI, 0.44 to 0.74 with p-value $<0.0001$; heterogeneity $I^{2}=72 \%$ with p-value $<0.01$ ) (Fig. 2A). Interestingly, in urine samples, hsa-miR-126 family expression in case groups was upregulated compared to control groups (OR 2931.12; 95\% CI, 9.96 to 862623.21 with p-value $=0.0059$; heterogeneity $I^{2}=97 \%$ with p-value $<0.01$ ) (see online suppl. ma-

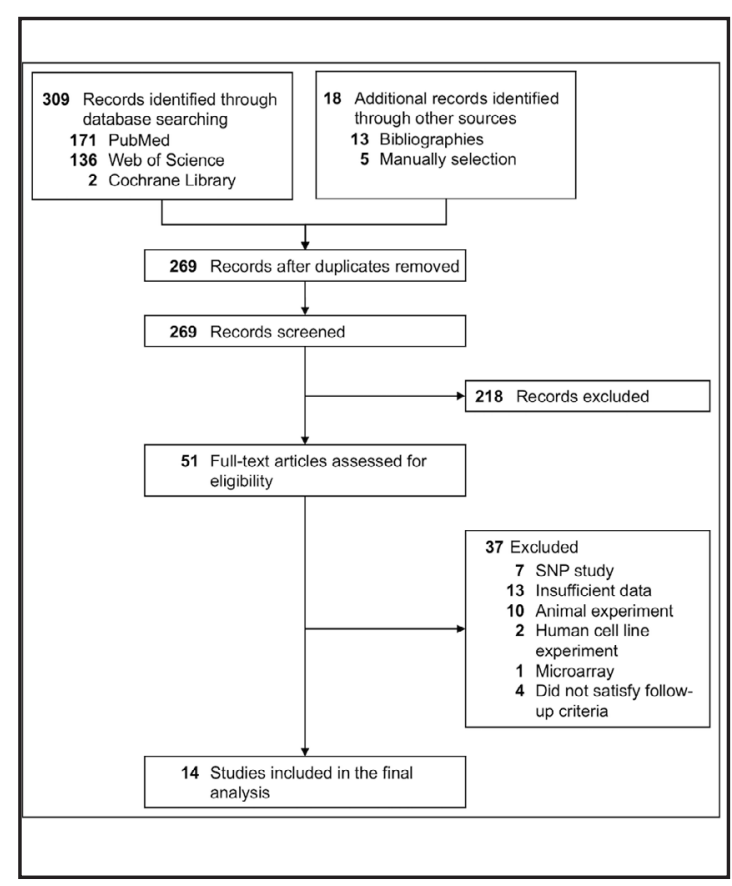

Fig. 1. Study Flow Diagram. Selection of clinical studies for meta-analyses of associations between DN and blood or urinary microRNAs. terial, Suppl. Fig. S2A).

Fig. 2. Meta-analyses: significant associations between microRNAs and DN. Hsa-miR-126 family in blood (A) and hsa-miR-770 family (B) were significantly associated with DN. The odds ratios (ORs) are represented within squares, and horizontal bars

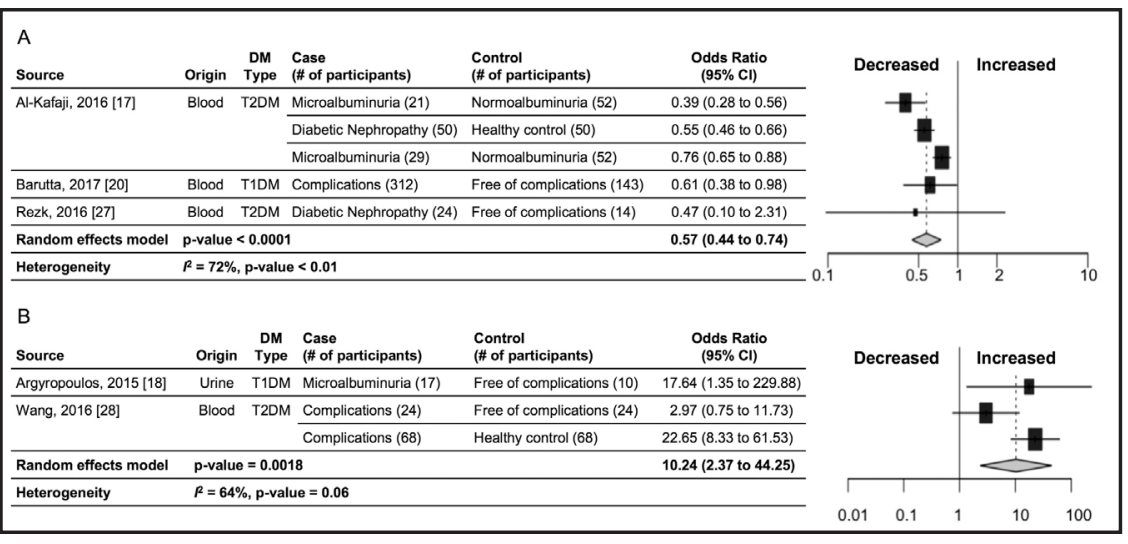
show 95\% confi-

dence intervals. This analysis gave pooled effects of OR 0.57 (95\% CI, 0.44 to 0.74 with p-value $<0.0001)$ for hsa-miR-126 in blood, and OR 10.24 (95\% CI, 2.37 to 44.25 with p-value $=0.0018$ ) for hsa-miR-770. "Complications" meant diabetic microvascular complications. "Free of complications" meant a diabetes mellitus patient who was observed to have no diabetic microvascular complications. 


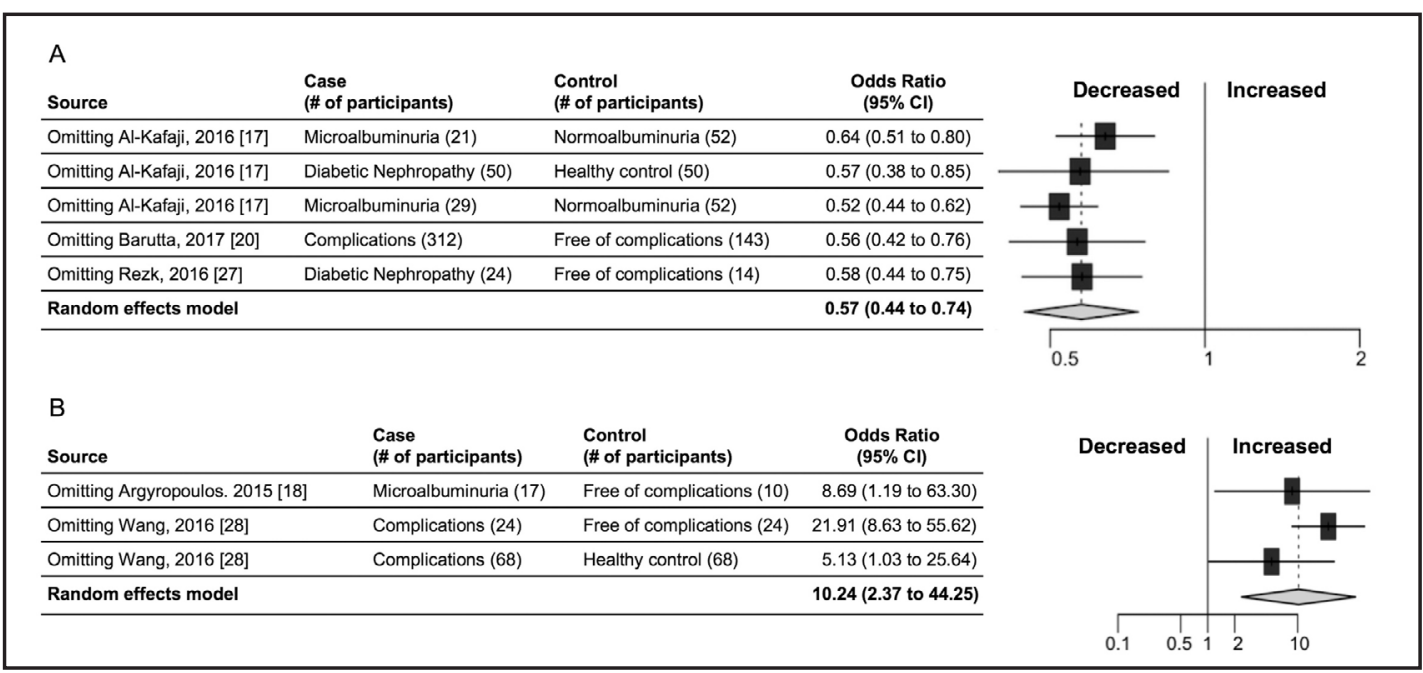

Fig. 3. One-study-removed sensitivity analyses of microRNA expression levels. (A) The OR of hsa-miR-126 in blood ranged from 0.52 to 0.64 in sensitivity analysis. (B) The OR of hsa-miR-770 ranged from 5.13 to 21.91 in sensitivity analysis.

Another highly significant microRNA we identified was the hsa-miR-770 family. It was upregulated in cases compared to controls (OR 10.24; 95\% CI, 2.37 to 44.25 with p-value = 0.0018; heterogeneity $I^{2}=64 \%$ with p-value $=0.06$ ) (Fig. 2B).

\section{Sensitivity Analysis and Publication Bias}

For our one-study-removed sensitivity analyses, we measured the effects of individual studies on the findings for miR-126 and miR-770 family expression (see previous section). This "sensitivity analysis" (Fig. 3 and (see online suppl. material) Suppl. Fig. S2B) indicated that individual studies did not change the conclusions.

We also visually inspected publication biases for the DN study models, as shown in (see online suppl. material) Suppl. Fig. S3. Here, the visual examination of funnel plots showed that all microRNA families' expression levels were not symmetric, implying publication bias. Consequently, we applied a "trim-and-fill" method [31] to estimate the number of potentially missing studies in our meta-analysis. In this method, the random-effects model of the hsa-miR-126 family in blood samples was slightly changed (OR: 0.65; 95\% CI: 0.5-0.84 with p-value $=0.001$; heterogeneity $I^{2}=74 \%$ with p-value $\left.=0.0008\right)$, with two additional nominal studies. This result indicates that the conclusion of Fig. $2 \mathrm{~A}$ was robust with regard to publication bias. We also note that due to the small number of studies, the trim-and-fill method was not applied to Fig. 2B and (see online suppl. material) Suppl. Fig. S2A.

\section{Discussion}

We meta-analyzed 14 selected clinical studies that had reported an association between circulating or urinary microRNAs and DN [16-29]. Our analysis demonstrated significant associations between DN and two microRNAs (hsa-miR-126 and hsa-miR-770 families). In the hsa-miR-126 family, different expression patterns were observed according to the sample origin (Fig. 2A, and Supplementary Fig. S2A). Blood hsa-miR-126 expression was lower in DN patients than in control groups (non-DN patients), whereas urinary hsa-miR-126 family expression was higher in case groups compared to control groups. These findings could be explained by positing that a loss of kidney resorption, and subsequent excretion, could deplete the circulating level of has-miR-126. For the hsa-miR-770 family (Fig. 2B), the same expression patterns were observed between the two sample types, i.e., upregulation in the case groups over the control groups. Although there have been some reports of association 


\section{Cellular Physiology Cell Physiol Biochem 2018;46:1331-1340

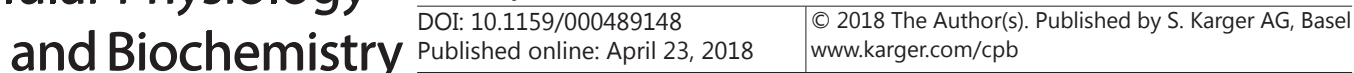 \\ Park et al.: A Meta-Analysis of MiRNAs in Diabetic Nephropathy}

between DN and the let-7 family, which are also involved in insulin sensitivity [34], we could not find an association between the let-7 family and DN (see online suppl. material, Suppl. Fig. S4). Additionally, looking into hsa-miR-221, hsa-miR-424, and hsa-miR-192, we did not find any associations with DN (see online suppl. material, Suppl. Fig. S5).

In (see online suppl. material) Suppl. Fig. S2A, the 95\% CI spanned a wide range, due to usages of different internal controls. In the Liu et al. study [24], PCR cycles of internal control were arbitrarily set to 50. Other studies, however, used small non-coding RNAs as internal controls for microRNA expression measurements.

Hsa-miR-126 plays an important role in maintaining endothelial cells, in which it enhances vascular endothelial growth factor (VEGF) signaling [35]. Circulating miR126 level has been negatively correlated with fasting blood glucose and hemoglobin A1c [27]. Interestingly, in contrast to blood miR-126, our meta-analyses showed that urinary miR-126 excretion was significantly increased in patients with DN, although there was a significant heterogeneity. This discrepancy may indicate either a local kidney-specific role or compensatory increases in VEGF and miR-126 in the pathogenesis and/or progression of DN involving microvascular endothelial cells [36, 37].

Although hsa-miR-770 has often been associated with diabetes and DN [28, 37-39], its precise function in diabetes complications remains unclear. Based on its targeting of the nucleotide excision repair enzyme ERCC2 in chemoresistant ovarian cancer $[40,41]$ and its sequestration by the upregulated IncRNA PCGEM1 in osteoarthritic synoviocytes [42], one could hypothesize an overall role in apoptosis. Further study is needed to elucidate the molecular mechanisms of miR-770 action in relation with DN.

This meta-analysis has some limitations. Although the number of subjects was more than 2,700 , studies inspecting the same microRNAs were relatively limited, so each aspect of our meta-analysis included a relatively small number of studies. The specific quantification methods of qPCR experiments also differed between studies, and the methods may affect the effect sizes. In fact, microRNA expression quantified in a study by Liu et al. [24] resulted in excessive effect sizes (see online suppl. material, Suppl. Fig. S2A). Additionally, despite a significant increase in hsa-miR-126 in the urine from DN patients (see online suppl. material, Suppl. Fig. 2A), the number of studies evaluating urine hsa-miR-126 was small. Another concern on effect measurement in our study could be the inclusion of urinary exosomal miRNAs. Free urinary miRNAs might also originate from the circulation and are confounded by plasma miRNAs which are able to pass the glomerular filtration barrier due to their small molecular weight. Urinary exosomal miRNAs most likely derived from different nephron segments as exosome size is greater than the cut-off size for glomerular filtration even in patients with macroalbuminuria. Therefore, the results should be carefully interpreted. To address these limitations, DN-related analyses of these microRNAs (miR-126 and miR770) need further investigation, using REMARK guidelines for biomarker translation [43]. Nonetheless, it has now become apparent that small, noncoding RNAs, such as microRNAs, will be reproducible, stable, and accurate biomarkers for numerous human maladies, including life-threatening diabetic nephropathy.

\section{Conclusion}

MiRNAs are novel non-invasive markers, indicating promising tools for the mechanistic investigation of diabetic renal pathophysiology (e.g., disease progression) and for potentially enabling us to monitor treatment effects. In near future, there will be more robust and convincing data. One issue is the normalization of data sets. In particular, the selection of an optimal housekeeper (urinary housekeeping miRNA) is a big hurdle, which complicates data comparison of qPCR data. Taken together, this meta-analysis is certainly the first step to get an overview of blood and urinary miRNA expression (miR-126 and miR-770 families) results from studies conducted so far. Nevertheless, miRNA data derived from urinary (exosomal) miRNA expression analyses are still too sparse to make any conclusions. 


\section{Cellular Physiology Cell Physiol Biochem 2018;46:1331-1340

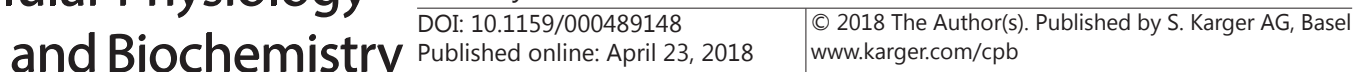 \\ Published onlıne: April 23, 1 A Meta-Analysis of MiRNAs in Diabetic Nephropathy}

\section{Acknowledgements}

The authors thank Dr. Curt Balch for editing the manuscript.

DHL conceived the meta-analysis design. DHL and SN supervised the study. SP performed the statistical analysis. SP, IBP, DHL and SN performed systematic inspection of publications for the meta-analysis. SM and KL assisted in the literature search. SP, DHL, and SN wrote the draft.

This study was supported by funding by the Korean Health Technology R\&D Project, through the Korean Health Industry Development Institute (KHIDI), funded by the Ministry of Health \& Welfare, Korea (HI16C1997) (to DHL).

\section{Disclosure Statement}

No conflict of interests exists.

\section{References}

1 Blair M: Diabetes Mellitus Review. Urol Nurs 2016;36:27-36.

-2 Ogurtsova K, da Rocha Fernandes JD, Huang Y, Linnenkamp U, Guariguata L, Cho NH, Cavan D, Shaw JE, Makaroff LE: IDF Diabetes Atlas: Global estimates for the prevalence of diabetes for 2015 and 2040. Diabetes Res Clin Pract 2017;128:40-50.

- Z Zimmet P, Alberti KG, Magliano DJ, Bennett PH: Diabetes mellitus statistics on prevalence and mortality: facts and fallacies. Nat Rev Endocrinol 2016;12:616-622.

-4 Saran R, Li Y, Robinson B, Abbott KC, Agodoa LY, Ayanian J, Bragg-Gresham J, Balkrishnan R, Chen JL, Cope E, Eggers PW, Gillen D, Gipson D, Hailpern SM, Hall YN, He K, Herman W, Heung M, Hirth RA, Hutton D, Jacobsen SJ, Kalantar-Zadeh K, Kovesdy CP, Lu Y, Molnar MZ, Morgenstern H, Nallamothu B, Nguyen DV, O’Hare AM, Plattner B, Pisoni R, Port FK, Rao P, Rhee CM, Sakhuja A, Schaubel DE, Selewski DT, Shahinian V, Sim JJ, Song P, Streja E, Kurella Tamura M, Tentori F, White S, Woodside K, Hirth RA: US Renal Data System 2015 Annual Data Report: Epidemiology of Kidney Disease in the United States. Am J Kidney Dis 2016;67:Svii, S1-305.

5 Afkarian M, Sachs MC, Kestenbaum B, Hirsch IB, Tuttle KR, Himmelfarb J, de Boer IH: Kidney disease and increased mortality risk in type 2 diabetes. J Am Soc Nephrol 2013;24:302-308.

6 Vupputuri S, Kimes TM, Calloway MO, Christian JB, Bruhn D, Martin AA, Nichols GA: The economic burden of progressive chronic kidney disease among patients with type 2 diabetes. J Diabetes Complications 2014;28:10-16.

7 Keane WF, Brenner BM, de Zeeuw D, Grunfeld JP, McGill J, Mitch WE, Ribeiro AB, Shahinfar S, Simpson RL, Snapinn SM, Toto R, Investigators RS: The risk of developing end-stage renal disease in patients with type 2 diabetes and nephropathy: the RENAAL study. Kidney Int 2003;63:1499-1507.

$>8$ Guay C, Regazzi R: Circulating microRNAs as novel biomarkers for diabetes mellitus. Nat Rev Endocrinol 2013;9:513-521.

-9 Sebastiani G, Nigi L, Grieco GE, Mancarella F, Ventriglia G, Dotta F: Circulating microRNAs and diabetes mellitus: a novel tool for disease prediction, diagnosis, and staging? J Endocrinol Invest 2017;40:591-610.

10 Wu J, Shao X, Lu K, Zhou J, Ren M, Xie X, Liu J, Xu Y, Ding Y, Shen X, Zhu C: Urinary RBP and NGAL Levels are Associated with Nephropathy in Patients with Type 2 Diabetes. Cell Physiol Biochem 2017;42:594-602.

-11 Agarwal R, Duffin KL, Laska DA, Voelker JR, Breyer MD, Mitchell PG: A prospective study of multiple protein biomarkers to predict progression in diabetic chronic kidney disease. Nephrol Dial Transplant 2014;29:2293-2302.

12 Trionfini P, Benigni A, Remuzzi G: MicroRNAs in kidney physiology and disease. Nat Rev Nephrol 2015;11:23-33.

13 Eissa S, Matboli M, Bekhet MM: Clinical verification of a novel urinary microRNA panal: 133b, -342 and -30 as biomarkers for diabetic nephropathy identified by bioinformatics analysis. Biomed Pharmacother 2016;83:92-99. 


\section{Cellular Physiology Cell Physiol Biochem 2018;46:1331-1340 \begin{tabular}{l|l} 
and Biochemistry Published 10.1159/000489148 & $\begin{array}{l}\text { D } 2018 \text { The Author(s). Published by S. Karger AG, Basel } \\
\text { www.karger.com/cpb }\end{array}$
\end{tabular}

14 Lv LL, Cao YH, Ni HF, Xu M, Liu D, Liu H, Chen PS, Liu BC: MicroRNA-29c in urinary exosome/microvesicle as a biomarker of renal fibrosis. Am J Physiol Renal Physiol 2013;305:F1220-1227.

15 Delic D, Eisele C, Schmid R, Baum P, Wiech F, Gerl M, Zimdahl H, Pullen SS, Urquhart R: Urinary Exosomal miRNA Signature in Type II Diabetic Nephropathy Patients. PLoS One 2016;11:e0150154.

-16 Barutta F, Tricarico M, Corbelli A, Annaratone L, Pinach S, Grimaldi S, Bruno G, Cimino D, Taverna D, Deregibus MC, Rastaldi MP, Perin PC, Gruden G: Urinary exosomal microRNAs in incipient diabetic nephropathy. PLoS One 2013;8:e73798.

17 Al-Kafaji G, Al-Mahroos G, Al-Muhtaresh HA, Skrypnyk C, Sabry MA, Ramadan AR: Decreased expression of circulating microRNA-126 in patients with type 2 diabetic nephropathy: A potential blood-based biomarker. Exp Ther Med 2016;12:815-822.

18 Argyropoulos C, Wang K, Bernardo J, Ellis D, Orchard T, Galas D, Johnson JP: Urinary MicroRNA Profiling Predicts the Development of Microalbuminuria in Patients with Type 1 Diabetes. J Clin Med 2015;4:14981517.

19 Argyropoulos C, Wang K, McClarty S, Huang D, Bernardo J, Ellis D, Orchard T, Galas D, Johnson J: Urinary microRNA profiling in the nephropathy of type 1 diabetes. PLoS One 2013;8:e54662.

20 Barutta F, Bruno G, Matullo G, Chaturvedi N, Grimaldi S, Schalkwijk C, Stehouwer CD, Fuller JH, Gruden G: MicroRNA-126 and micro-/macrovascular complications of type 1 diabetes in the EURODIAB Prospective Complications Study. Acta Diabetol 2017;54:133-139.

-21 Bijkerk R, Duijs JM, Khairoun M, Ter Horst CJ, van der Pol P, Mallat MJ, Rotmans JI, de Vries AP, de Koning EJ, de Fijter JW, Rabelink TJ, van Zonneveld AJ, Reinders ME: Circulating microRNAs associate with diabetic nephropathy and systemic microvascular damage and normalize after simultaneous pancreas-kidney transplantation. Am J Transplant 2015;15:1081-1090.

-22 Garcia de la Torre N, Fernandez-Durango R, Gomez R, Fuentes M, Roldan-Pallares M, Donate J, Barabash A, Alonso B, Runkle I, Duran A, Rubio MA, Calle-Pascual AL: Expression of Angiogenic MicroRNAs in Endothelial Progenitor Cells From Type 1 Diabetic Patients With and Without Diabetic Retinopathy. Invest Ophthalmol Vis Sci 2015;56:4090-4098.

-23 Jia Y, Guan M, Zheng Z, Zhang Q, Tang C, Xu W, Xiao Z, Wang L, Xue Y: miRNAs in Urine Extracellular Vesicles as Predictors of Early-Stage Diabetic Nephropathy. J Diabetes Res 2016;2016:7932765.

24 Liu Y, Gao G, Yang C, Zhou K, Shen B, Liang H, Jiang X: Stability of miR-126 in Urine and Its Potential as a Biomarker for Renal Endothelial Injury with Diabetic Nephropathy. Int J Endocrinol 2014;2014:393109.

25 Ma X, Lu C, Lv C, Wu C, Wang Q: The Expression of miR-192 and Its Significance in Diabetic Nephropathy Patients with Different Urine Albumin Creatinine Ratio. J Diabetes Res 2016;2016:6789402.

26 Pezzolesi MG, Satake E, McDonnell KP, Major M, Smiles AM, Krolewski AS: Circulating TGF-beta1-Regulated miRNAs and the Risk of Rapid Progression to ESRD in Type 1 Diabetes. Diabetes 2015;64:3285-3293.

27 Rezk NA, Sabbah NA, Saad MS: Role of MicroRNA 126 in screening, diagnosis, and prognosis of diabetic patients in Egypt. IUBMB Life 2016;68:452-458.

28 Wang C, Wan S, Yang T, Niu D, Zhang A, Yang C, Cai J, Wu J, Song J, Zhang CY, Zhang C, Wang J: Increased serum microRNAs are closely associated with the presence of microvascular complications in type 2 diabetes mellitus. Sci Rep 2016;6:20032.

29 Zampetaki A, Willeit P, Burr S, Yin X, Langley SR, Kiechl S, Klein R, Rossing P, Chaturvedi N, Mayr M: Angiogenic microRNAs Linked to Incidence and Progression of Diabetic Retinopathy in Type 1 Diabetes. Diabetes 2016;65:216-227.

30 Higgins J, Green S: Cochrane Handbook for Systematic Reviews of Interventions. Version 5.1.0. Updated March 2011.,

31 Duval S, Tweedie R: Trim and fill: A simple funnel-plot-based method of testing and adjusting for publication bias in meta-analysis. Biometrics 2000;56:455-463.

32 Schwarzer G: meta: An R package for meta-analysis. R News 2007; 7:40-45.

-33 Livak KJ, Schmittgen TD: Analysis of relative gene expression data using real-time quantitative PCR and the 2(-Delta Delta C(T)) Method. Methods 2001;25:402-408.

-34 Frost RJ, Olson EN: Control of glucose homeostasis and insulin sensitivity by the Let-7 family of microRNAs. Proc Natl Acad Sci U S A 2011;108:21075-21080.

-35 Wang S, Aurora AB, Johnson BA, Qi X, McAnally J, Hill JA, Richardson JA, Bassel-Duby R, Olson EN: The endothelial-specific microRNA miR-126 governs vascular integrity and angiogenesis. Dev Cell 2008;15:261-271. 


\section{Cellular Physiology Cell Physiol Biochem 2018;46:1331-1340 and Biochemistry Published online:April 23, $2018 \quad \begin{aligned} & \text { DOI 10159/2018 The Author(s). Published by S. Karger AG, Basel } \\ & \text { www.karger.com/cpb }\end{aligned}$ \\ Park et al.: A Meta-Analysis of MiRNAs in Diabetic Nephropathy}

36 Prattichizzo F, Giuliani A, Ceka A, Rippo MR, Bonfigli AR, Testa R, Procopio AD, Olivieri F: Epigenetic mechanisms of endothelial dysfunction in type 2 diabetes. Clin Epigenetics 2015;7:56.

-37 Prattichizzo F, Giuliani A, De Nigris V, Pujadas G, Ceka A, La Sala L, Genovese S, Testa R, Procopio AD, Olivieri F, Ceriello A: Extracellular microRNAs and endothelial hyperglycaemic memory: a therapeutic opportunity? Diabetes Obes Metab 2016;18:855-867.

-38 Ghai V, Wang K: Recent progress toward the use of circulating microRNAs as clinical biomarkers. Arch Toxicol 2016;90:2959-2978.

39 Wu B, Miller D: Involvement of MicroRNAs in Diabetes and Its Complications. Methods Mol Biol 2017;1617:225-239.

40 Steffensen KD, Waldstrom M, Jakobsen A: The relationship of platinum resistance and ERCC1 protein expression in epithelial ovarian cancer. Int J Gynecol Cancer 2009;19:820-825.

41 Zhao H, Yu X, Ding Y, Zhao J, Wang G, Wu X, Jiang J, Peng C, Guo GZ, Cui S: MiR-770-5p inhibits cisplatin chemoresistance in human ovarian cancer by targeting ERCC2. Oncotarget 2016;7:53254-53268.

42 Kang Y, Song J, Kim D, Ahn C, Park S, Chun CH, Jin EJ: PCGEM1 stimulates proliferation of osteoarthritic synoviocytes by acting as a sponge for miR-770. J Orthop Res 2016;34:412-418.

43 Dancey JE, Dobbin KK, Groshen S, Jessup JM, Hruszkewycz AH, Koehler M, Parchment R, Ratain MJ, Shankar LK, Stadler WM, True LD, Gravell A, Grever MR, Biomarkers Task Force of the NCIIDSC: Guidelines for the development and incorporation of biomarker studies in early clinical trials of novel agents. Clin Cancer Res 2010;16:1745-1755. 